\title{
An Alternative to an Artificial Hand
}

\author{
By MISS J. McGUIRE, M.C.S.P.,
}

Jane Furse Hospital, Sekhukhuniland, Transvaal

For the African patient who has to have an amputation of hand, an artificial limb is not always practicable, especially in the Reserve area. A usclul alternative has been found in the Krukenberg's operation. This divides the radius and ulna into separate and mobile "jaws" and so provides an effective pincer-like grip.

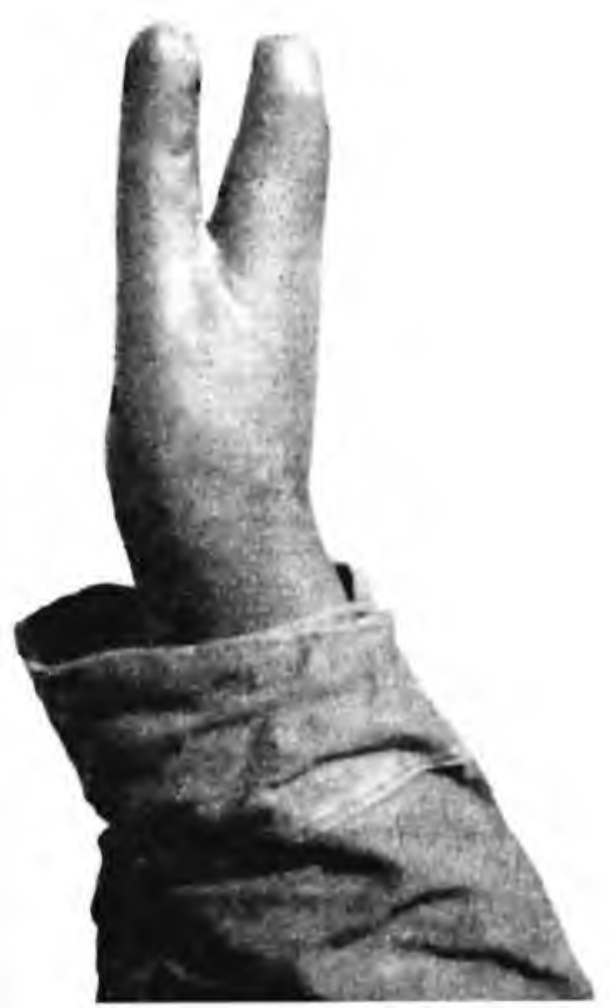

\section{Case History}

Jacobus, an African aged 35, suffered severe burns of both hands during an epileptic fit in April, 1963. His right hand had been amputated $5 \mathrm{~cm}$. above the wrist-joint, and the left hand had been severely mained. He was first seen here six months later, when he was complaining of a partial skin breakdown of the left hand. He had at that time virtually no movement in the wrist or hand, the metacarpophalangeal joints being hyper-extended and the phalangeal ones flexed. Together with the stump on the right arm any self-help was severely limited, and so the Krukenberg's operation was decided upon.
With this in view, resisted movements were encouraged for the elbow and shoulder of the right arm, and particular attention was paid to pro and supination of the forearm. At the same time, I tried to obtain what movement was possible in the left hand, doing the exercises initially in a saline bath. I padded a spoon handle, and Jacobus gripped it between thumb and first metacarpel-phalangeal joint, and was thus able to feed himself again.

\section{Operation Notes}

Flexol digitorum sublimis was divided into two halves and the interosseous membrane divided; the extensor muscles were divided into two groups. A large neuroma on the end of the median nerve was removed, and the nerve shortened. Flexor digitorium profundis and flexor hallucis longus were dissected out and removed, and the belly of extensor digitorium was also removed. Extensors and flexors on the radial half were well attached to the end of the bone by scar tissue. The flexor tendons were slack and needed shortening. The skin was closed over the radial digit, but had to have a skin graft shortly after over the ulna, as there was not enough skin to cover the inside of both radius and ulna. Dr. Nollidge said he was greatly aided by the fact that the amputation was well-healed, with already shrunken, though healthy, forearm muscles.

\section{Treatment}

Within a few days, Jacobus was ablc to gently abduct the radius. After a month, he could open and close the "pincers" quickly, grip a soft rubber wedge firmly, and was picking light objects out of a box and putting them on the table.

The muscle work involved was interesting. To open the "hand" he used pronator teres, helped by brachio-radialis and possibly extensor carpi radialis. To close the hand and grip he used supinator, helped by biceps, and both movements could be done with the elbow either flexed or extended. Before discharge several months later, the "jaws" could be opened to an angle of $23^{\circ}$ (i.e. a distance of about 2 inches between the bone ends). Once the tenderness over the bone ends had gone, the patient had a grip that was considerably stronger than the one exerted between the normal thumb and first metacarpel; it can be expected to increase still more.

He could, by using a grip between the knuckles of his left hand and the 'pincer', completely lace up a shoe and tie a bow. With the 'pincer' only he could feed himself with a padded spoon and hold things like an average sized book. He was able to pick up objects as small as a match, and could pick up and hurl a $2 \mathrm{lb}$. sandbag from one end of the room to the other! The latter took time to learn, as the 'pincer' refused to release at the exact moment required at first. Had Jacobus been literate, he would have been able to write with a V-shaped wooden pencilholder. At his discharge he was supplied with trousers with a zip fastener, as small buttons still defeated him.

I would like to thank Dr. Nottidge for the use of his notes on the case, and for his co-operation throughout. 